Invited Paper

\title{
Next generation of optical fibre sensors: new concepts and perspectives
}

\author{
Luc Thévenaz \\ EPFL Swiss Federal Institute of Technology, Group for Fiber Optics (GFO), \\ SCI-STI-LT, Station 11, CH-1015 Lausanne, Switzerland \\ E-mail: luc.thevenaz@epfl.ch
}

\begin{abstract}
Fibre sensing has reached an interesting turning point today: a clearer assessment about promises that were really held and those that failed to convince can be envisioned. A choice of key fibre sensing techniques will be reviewed and a critical discussion about their real impact will be presented. The promising potentialities of distributed sensing in its different flavours will be addressed, without omitting perspectives on other major techniques, such as Bragg gratings and chemical sensors.
\end{abstract}

Keywords: optical fibre sensor, distributed fibre sensor, fibre Bragg grating, optical reflectometry

\section{INTRODUCTION}

Optical fibre sensing is one of these typical and exemplary fields of research that require a deep scientific search for novel concepts and solutions, based on a rich and diverse physical background, but are definitely slowly maturing by showing no flashing growth that would attract the attention from the public and the big investors. Since the technology has now come to age, a quick look back over the shoulder shows that a modest but solid commercial vitality remains mostly restricted to fibre gyroscopes, to sensor system based on fibre Bragg gratings (FBG) and of course to distributed fibre sensors that particularly benefit from the physical geometry and optical properties of the silica fibre.

The intention behind this paper is definitely not to present a review of the state-of-the-art and to allocate good and bad marks to research works and to practical realizations. This would be in all cases non-exhaustive and could be unintentionally experienced like offending by colleagues having invested efforts with a sincere conviction. Scientific rigour also prevents from making predictions for the future, since it is by essence unpredictable, and a natural confidence in the human creativity supports a personal conviction that novel discoveries and smart solutions will thwart the certainties of the best oracles.

Instead, existing approaches will be critically analysed in this paper, with the ambition to determine in which directions a progress can still be foreseen, and also unfortunately in what directions present efforts are likely to be vain. This will be addressed by placing the focus on aspects that are often strategically omitted in publications. This exercise will be limited to the 2 currently growing field of research in optical fibre sensing: chemical-biological and distributed sensing, keeping in mind that the same analysis could be performed for other types of fibre sensing.

\section{CHEMICAL SENSING}

It is important to keep in mind the finality of a chemical sensor, which is to detect the presence and to evaluate the concentration of a given molecular species, normally highly dissolved in a fluid, which can simply be the ambient air for environmental sensing. A chemical sensor must therefore be designed to best fulfil these operating conditions:

- $\quad$ it must be sensitive to detect molecules in very low concentration (from ppm down to ppb or less);

- it must be highly selective to solely respond to a specific molecule species, without interfering response from other molecules even at very high concentration;

- the response must only depend on the molecular concentration, with no influence from other environmental quantities (such as temperature) and from the molecular content of the dissolving solution.

A sensor satisfying all these conditions may be considered as perfect; however, perfection does not belong to this world and practical sensors do not normally fully realize all these conditions. The class of chemical sensors that best fit these conditions is those based on spectroscopic measurements: each molecule presents spectral absorption lines at welldefined wavelengths that are like a fingerprint of this species. It is an evidence that optics has a key role to play in

23rd International Conference on Optical Fibre Sensors, edited by José Miguel López-Higuera,

Julian Jones, Manuel López-Amo, José Luis Santos, Proc. of SPIE Vol. 9157, 9157AN

(C) 2014 SPIE $\cdot$ CCC code: $0277-786 X / 14 / \$ 18 \cdot$ doi: $10.1117 / 12.2064896$ 
spectroscopic sensing. By selectively measuring the absorption at one (or several) of these wavelengths, the sole concentration of this molecule can be in principle measured. Practically, since the absorption lines are not infinitely narrow and spread over several $\mathrm{GHz}$ in normal atmosphere, the interference of close absorption lines from other species at much higher concentrations cannot be entirely avoided. In normal atmosphere water vapour is highly concentrated and shows a very rich and complex absorption spectrum, with spectral lines covering nearly all the spectral regions of interest. In this case, even extremely weak $\mathrm{H}_{2} \mathrm{O}$ absorption lines are problematic, since they can eventually show comparable absorptions to intense absorption lines of the molecule of interest at sub-ppm concentration.

Before investing big efforts to seek for fibre-based solutions, it is also important to keep in mind that there is a wide choice of robust and reliable chemical sensors based on classical techniques, which can measure concentrations down to ppm for the price of a portable audio player. So, except for particular molecules absolutely reluctant to the measurement by classical means, it looks like a lost cause to develop a fibre-based sensor for concentrations higher than $1 \mathrm{ppm}$.

Without being exhaustive some type of fibre-based sensors can be reviewed, keeping in mind the above listed properties that define an ideal chemical sensor. The focus of this brief analysis will be placed on the specific advantages that may be brought by optical fibres.

\section{Direct spectroscopic sensing}

In this configuration a beam of light is crossing the gaseous or liquid solution to be analysed and differential transmission is measured at multiple close wavelengths to determine the tiny absorption due to a molecular spectral line of the species to be measured. This approach looks poorly compatible with the light guiding in optical fibres, since light is essentially confined in the waveguide without direct contact with the analyte.

A possibility is to use hollow-core fibres, in which case the overlapping with the analyte will be perfect if the solution can circulate in the hollow core. This scheme turns out to be actually poorly effective, since the fibre core is of micrometric size and even a very low viscosity of the solution prohibits an effective circulation of the analyte. It was proposed to drill lateral holes in the fibre to let the solution enter the fibre core more easily, but this solution requires a fibre machining and large holes to be efficient.

Another approach is to use the evanescent field of a micro-fibre (silica wire), but this solution turns out to present several irreducible difficulties: the fraction of optical power in the evanescent field exceeds $10 \%$ only when the micro-fibre diameter is smaller than 1 micron, requiring very long fibre lengths to obtain sensitive absorptions and such lengths are impractical in nano-fibres. In addition the fibre core is laterally exposed to the environment, in particular dust, and rapidly huge attenuations are observed.

Fibre could bring an original solution to realize distributed chemical sensing, but such a solution is also subject to the same practical penalties presented here above, so that fibres do not look like bringing decisive advantages for direct spectroscopic sensing.

\section{Refractive index sensing}

Several implementations have been recently proposed to achieve extremely sensitive measurement of the refractive index of a liquid solution. Any change of the chemical content of a solution will modify the refractive index, in particular any change of concentration of the target molecule in the solution. Extreme sensitivities have been obtained, with measured changes of the order of $10^{-7}$ in the refractive index value, which are compatible with the detection of subppm concentration changes. Most implementations are based on resonant couplings, using Bragg gratings, long period gratings, cavities and plasmons.

A quick analysis shows that the refractive sensing actually only fulfils the condition of sub-ppm sensitivity, but is not at all selective and is highly subject to environmental changes, in particular temperature and pressure. Any change in the molecular concentration of any species in the analyte will equally impact on the refractive index. And the effect of temperature is even larger: in the most common liquid, i.e. water, the refractive index change is approximately $10^{-3}$ per degree, requiring a thermal stabilization down to the milliKelvin for a ppm sensitivity. It is easy to conclude that refractive index sensing will probably face incommensurable difficulties to be a convincing chemical sensor.

\section{Indirect chemical sensing}

Interesting smart solutions based on optical fibres have been proposed these past few years, some of them very imaginative, and it can be predicted that solutions based on indirect sensing have probably the largest probability of 
success. Many are based on the micromachining of the tip of optical fibres to realize micro resonators, acoustic sensors, photoacoustic microcavities, micro-evaporators, etc... In this case the fibre is essentially used to convey the light up to the fibre tip, where the interaction between light and the chemical solution takes place. This interaction may induce fluorescence, a quick and transient temperature or pressure change, or even simply monitoring the volume change of a solution droplet due to evaporation, and this can be monitored by a signature on the returned light. Since the interaction with light is confined to the fibre tip only, the operation of the sensor is much less subject to perturbation and dust, while securing a direct access to the analyte and a fast response.

\section{DISTRIBUTED FIBRE SENSING}

Distributed optical fibre sensing is such a fascinating technique that most efforts have been focussed on the realization of record performance ${ }^{1,2}$ and no attention has been enough paid to the analysis of the best conditions to be realized to get an optimized sensing response. It may be helpful at this point to remind the important parameters and conditions to be optimized for best distributed fibre sensing:

I. The primary key parameter is the signal-to-noise ratio (SNR) on the raw response (temporal trace or equivalent). This sounds like an evidence, but only recent research works have been focussed on the optimization of this parameter, with quantitative evaluation on its impact on the final response ${ }^{3}$. Improving the SNR universally benefits to any performance of the sensor: longer distance range, sharper spatial resolution, better accuracy on the locally measured quantity and finally faster measurement through reduced averaging.

II. The next important parameter is the sensitivity of the response to the measured quantity. Currently quantities intrinsically measured by fibre sensors are restricted to temperature, strain and birefringence. Except for Raman-based sensing, in all distributed sensing techniques the information on the changes in the measured quantity is obtained by the determination of a resonance shift (or correlation shift) in the optical frequency domain. For instance, for temperature sensing, this sensitivity is expressed in $\mathrm{Hz}$ of resonance shift per degree of temperature change. The most sensitive dependence occurs when the resonance shift is function of the refractive index change due to temperature, which globally amounts to $\sim 1.3 \mathrm{GHz} / \mathrm{K}$ on the resonance shift. This parameter also impacts on all properties of the sensor, since for a given target accuracy on the measured quantity a higher sensitivity requires a smaller SNR.

III. The last important parameter is the linear depletion due to the interaction, which must remain small enough to prevent from an excessive cumulated depletion of the activating signal. A too large depletion leads to biased measurements when retrieving the information in the optical frequency domain ${ }^{4}$. A simple rule to avoid this problem is to design the sensor, so that the linear depletion keeps much smaller than the linear loss, or to realize conditions in which depletion is frequency-independent or compensated.

Now the distributed sensors can be classified into 2 broad categories:

A. Those based on spontaneous backscatterings, in which the activating signal (e.g. a light pulse in time-domain reflectometry) is isotropically scattered in all directions during its propagation all along the fibre. A small fraction of this scattered light is recaptured by the fibre core in the back direction and returned to the instrument for detection and

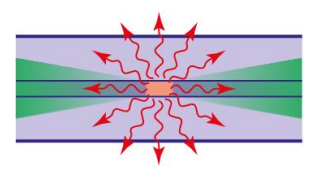
analysis. So this class of sensors suffer from an intrinsic penalty, since only a tiny fraction of the light depleted from the activating signal is eventually recaptured to build the sensor response. In standard fibre the recapture factor is of the order of $0.15 \%$, so that most of the scattered light is actually wasted and the intrinsic irreducible penalty on the signal intensity (and thus the SNR) is $\sim-28 \mathrm{~dB}$.

B. Those based on a coherent coupling through a resonant longitudinal structure such as a Bragg reflector. The fibre Bragg gratings enter into this category, but also all sensors based on a nonlinear wave coupling such as those based on stimulated scatterings. In ideal conditions (that are practically met) all light depleted from the activating signal is

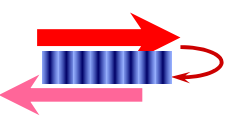
backcoupled and returned to the instrument, so that the interaction can be considered lossless or with a $0 \mathrm{~dB}$ penalty on the response.

Within this broad framework it is possible to analyse and to compare from a very general point of view the potentiality of the different existing distributed fibre sensors, roughly classified by growing performance. 
1. Raman-based sensors: In this pure intensity-coded and pure temperature sensing system the scattering crosssection is approximately $40 \mathrm{~dB}$ smaller than Rayleigh scattering. It means that condition III is largely satisfied, but the response amplitude is far from ideal. In addition it is subject to the recapture loss, so that the total penalty is approx. $\mathbf{6 8} \mathbf{~ d B}$ with respect to an ideally responding sensor. The change in intensity is approx. $0.6 \% / \mathrm{K}$, difficult to fairly compare with a resonance-based sensor, but all in all this type of sensor can be considered as offering a response which is the most distant to the ideal case.

2. Spontaneous Brillouin-based sensors (BOTDR): In this resonance-based sensor the scattering cross-section is approximately $20 \mathrm{~dB}$ smaller than Rayleigh scattering. The response amplitude is again not close to ideal and the total penalty is approx. $\mathbf{4 8} \mathbf{~ d B}$ including the recapturing factor. Besides, the resonance shift due to temperature is not ruled by refractive index changes, but by the acoustic velocity dependence on temperature that is $\sim 1 \mathrm{MHz} / \mathrm{K}$, corresponding to another $\mathbf{3 1} \mathbf{~ d B}$ penalty when compared to the ideal dependence on refractive index, resulting in a total penalty of $79 \mathbf{~ d B}$ with respect to an ideal response.

3. Stimulated Brillouin-based sensors (BOTDA): In this type of sensors a coherent coupling is realized, so that such a system can be intelligently designed to realize a perfect transfer while keeping within the limit given by condition III. Nevertheless the coherent coupling is activated by the interaction of 2 signals: a pump pulse and a $\mathrm{CW}$ probe wave. This $\mathrm{CW}$ probe is perfectly superposed to the sensor response, so that the response will be only a fraction of the total detected intensity. Considering that practically the dominating noise is relative intensity noise (RIN) this $\mathrm{CW}$ background will cause a penalty equal to the local interaction gain, which is of the order of $1 \%$, corresponding to a $\mathbf{2 0 ~ d B}$ penalty on the SNR. Since the resonance shift is subject to the same reduced response like the BOTDR $(\mathbf{3 1} \mathbf{d B})$, the total penalty amounts to $\mathbf{5 1} \mathbf{~ d B}$ for this type of sensors.

4. Coherent Rayleigh-based sensors (C-OTDR): Condition III is intrinsically satisfied in this type of sensors (frequency-independent interaction) and the resonance shift is ruled by refractive index changes, so that the penalty is just due to the recapturing factor of $28 \mathbf{~ d B}$.

5. Dynamic Brillouin gratings sensing (DBG): This type of sensor employs highly birefringent fibres and the dynamic writing of the gratings through SBS and the resonance reading are realized using orthogonal polarizations. Globally the situation is similar to the BOTDA (case 3), but no CW probe wave superimposes on the response, so that a $\mathbf{0} \mathbf{d B}$ SNR penalty is observed for the energy transfer from the activating signal to the response. The resonance shift is governed by the temperature dependence of the induced birefringence in the fibre which is normally higher than the acoustic velocity dependence. A value of $55 \mathrm{MHz} / \mathrm{K}$ is reported repat $^{5}$ may vary from fibre to fibre, which is $4 \%$ of the ideal refractive index dependence, giving a total 14 dB penalty.

6. Faint long gratings (FLOG): an ultra-weak fibre Bragg grating is assumed to be printed all along the sensing optical fibre. All the light is back coupled in this case ( $\mathbf{0} \mathbf{~ d B}$ penalty) and the resonance shift is governed by the refractive index change ( $\mathbf{0} \mathbf{~ d B}$ penalty), so that this should be the perfect distributed fibre sensor showing an ideal response. The massive practical hindering is related to the printing of a continuous faint grating with no phase hopping all along the fibre, which is yet far from being realized over large distance.

This analysis shows that substantial progresses can still be realized in distributed sensors by moving the focus on the optimization of the SNR. In a matter of less than 5 years distributed fibre sensors showing a 20-30 dB gain in their global response with respect to existing sensors will be very probably demonstrated, resulting in equal gain in performance. This will make distributed systems the flagships of fibre sensing and a key technology for the future.

\section{REFERENCES}

[1] Thévenaz, L., "Brillouin distributed time-domain sensing in optical fibers: state of the art and perspectives", Frontiers of Optoelectronics in China 3(1), 13-21 (2010).

[2] Bao, X. and Chen, L., "Recent Progress in Brillouin Scattering Based Fiber Sensors", Sensors 11(4), 4152-4187 (2011).

[3] Soto, M. A. and Thévenaz, L., "Modeling and evaluating the performance of Brillouin distributed optical fiber sensors", Optics Express 21(25), 31347-31366 (2013).

[4] Thévenaz, L., Foaleng Mafang, S. and Lin, J., "Effect of pulse depletion in a Brillouin optical time-domain analysis system", Opt. Express 21(12), 14017-14035 (2013).

[5] Zou, W., He, Z. and Hotate, K., "Complete discrimination of strain and temperature using Brillouin frequency shift and birefringence in a polarization-maintaining fiber", Opt. Express 17(3), 1248-1255 (2009). 